\title{
Sampling analytes from cheese products for fast detection using neutral desorption extractive electrospray ionization mass spectrometry
}

\author{
Zhongchen Wu • Konstantin Chingin • Huanwen Chen • \\ Liang Zhu $\cdot$ Bin Jia $\cdot$ Renato Zenobi
}

Received: 22 January 2010 /Revised: 26 March 2010 / Accepted: 28 March 2010 /Published online: 23 April 2010

(C) Springer-Verlag 2010

\begin{abstract}
The development of analytical techniques suitable for sensitive, high-throughput, and nondestructive food analysis has been of increasing interest in recent years. In this study, mass-spectral fingerprints of various cheese products were rapidly recorded in the mass range of $\mathrm{m} / \mathrm{z} 50-300 \mathrm{Da}$ without any sample pretreatment, using neutral desorption extractive electrospray ionization mass spectrometry (NDEESI-MS) in negative ion mode. The results demonstrate that both volatile and nonvolatile analytes on greasy cheese surfaces can be directly sampled by a neutral desorption gas beam. The influence of the neutral desorption gas flow on the analyte signal was systematically investigated. Under optimized experimental conditions, reproducible results were obtained using ND-EESI-MS. Principal component analysis was applied to differentiate a total of 49 individual cheese samples (four different types), which were purchased from three different supermarkets. All samples were successfully
\end{abstract}

Electronic supplementary material The online version of this article (doi:10.1007/s00216-010-3693-9) contains supplementary material, which is available to authorized users.

Z. Wu $\cdot$ H. Chen $\cdot$ B. Jia

Department of Applied Chemistry,

East China Institute of Technology,

Fuzhou 344000, China

H. Chen

e-mail: chw8868@gmail.com

K. Chingin $\cdot$ L. Zhu $\cdot$ R. Zenobi $(\triangle)$

Department of Chemistry and Applied Biosciences, ETH Zurich,

HCI E329,

8093 Zurich, Switzerland

e-mail: zenobi@org.chem.ethz.ch

Z. Wu

School of Space Science and Physics,

Shandong University at Weihai,

Weihai 264209, China classified according to their types; but distributors and sensory properties were not distinguishable from the spectra data. The principal components 2, 3, and 4 scores showed an excellent capacity of distinguishing types of cheese. Molecular markers of interest can be identified using tandem mass spectrometry and matching the data with those from reference compounds. The experimental data show that ND-EESI-MS is able to sensitively and directly detect analytes on greasy surfaces without chemical contamination, providing a convenient method for high-throughput food analysis with a high degree of safety.

Keywords Neutral desorption extractive electrospray ionization · Cheese · Food safety · Greasy surfaces · Principal component analysis

\section{Introduction}

Contaminated food such as the recent melamine-tainted milk is a serious health hazard [1], and therefore the assurance of food safety compliance is very important for public health. Rapid food analysis represents an urgent need, but challenges modern analytical science in many aspects $[2,3]$, including sensitivity, selectivity, and sample throughput.

Cheeses are fermented milk-based food products, serving as an important daily protein source, especially for people in Europe and America. Cheese products are intrinsically complex mixtures, and the large number of steps required for their commercial production further complicates them $[4,5]$. Many factors, such as the source and quality of milk [6], animal feed [7], and manufacturing conditions [8], can affect the features of the final product, and play important roles in the formation of flavor and taste. There are nowadays more than 500 different types of cheese products 
in the world [9] with various morphological characters. All the above-mentioned factors highlight the practical needs for direct and fast cheese characterization.

Mass spectrometry (MS) has been recognized as the best "sensor" available for food analysis [10] owing to its superior sensitivity and selectivity. Recently developed ambient ionization techniques such as direct analysis in real time [11], matrix-assisted laser desorption electrospray ionization (MALDESI) [12], desorption ESI [13, 14], surface desorption atmospheric pressure chemical ionization [15, 16], and extractive ESI (EESI) [17-19] are possible choices for rapid analysis for complex foods such as cheese. These methods combine sensitive and specific detection with minimal or no sample pretreatment, which opens ways to high-throughput analyses of a wide range of samples using MS. Biological surfaces can be gently probed using neutral desorption (ND), allowing fast and sensitive characterization of complex biological surfaces when combined with EESI-MS (ND-EESI-MS) [20, 21]. By separating the sampling and ionization processes in both space and time [20, 22], ND-EESI tolerates extremely complex matrices, provides a high degree of safety of the experimental procedure, and avoids sample contamination. These figures of merit render ND-EESI-MS suitable for fast and safe analysis of greasy samples such as cheese.

The identification of food types is very important for commercial trade and safety management. Some analysis techniques coupled with chemometric methods were reported for identification of particular brands. For example, nearinfrared spectroscopy was applied to identify brands of milk powder [23], instant noodles [24], and injection cephalosporins [25]. Still, near-infrared spectral data give little information about the specific chemical composition, which is very important for quality assurance. An electronic nose was used for cigarette brand identification [26]. However, an electronic nose is designed only for odor detection based on the trained library of known fingerprints, which greatly restricts its applications [26]. Nuclear magnetic resonance spectroscopy was reported to differentiate the brands of beer products [27]. The large size of nuclear magnetic resonance instruments renders field applications of this technique problematic. Some mass-spectrometric techniques, such as isotope ratio MS [28] and proton transfer reaction MS [29], have been also applied successfully to identify the geographic origin of cheese products.

In this study, ND-EESI-MS was implemented with a commercial quadrupole time-of-flight (QTOF) mass spectrometer to directly characterize cheese products in negative ion mode to obtain characteristic mass fingerprints with a high signal-to-noise ratio. The negative ion mode was chosen because of the high content of fatty acids in cheese. Fatty acids are, of course, much more easily deprotonated than protonated. As a result, MS fingerprints in negative ion mode are much more meaningful than in positive ion mode. All the mass-spectral fingerprints were recorded from the greasy cheese surface and were used to classify cheese products according to their types. Our results show that ND-EESI-MS is able to sample both volatile and nonvolatile compounds from the greasy cheese surfaces, providing a useful and safe tool for rapid food analysis.

\section{Materials and methods}

Reagents and materials

Chemical reagents and solvents were bought from Fluka (Buchs, Switzerland) and were of the highest purity available for direct use without any further purification. Deionized water was produced by a Nanopure waterpurification system (Barnstead, Dubuque, IA, USA). Four types of commercial cheese products (a total of 49 individual samples) were randomly selected from three well-known supermarkets (Denner, Coop, and Migros) in Switzerland. All the cheeses were quality-guaranteed products with various expiration dates. Detailed information about the samples analyzed in this experiment is given in Table 1. All samples were produced in Switzerland using either raw or pasteurized cow's milk, but apart from taste were difficult to differentiate by sensory qualities such as color, texture, and hardness. The cheese samples were directly analyzed without further treatment at room temperature in open air.

\section{ND-EESI mass analysis}

A commercially available QTOF mass spectrometer (QTOF Ultima, Waters Micromass, Manchester, UK) was employed in this study. All mass spectra were background-subtracted by the operating software (MassLynx ${ }^{\circledR}$ version 4.01, Waters, Manchester, UK). For tandem MS studies, parent ions were isolated with single mass/charge unit width and collisioninduced dissociation (CID) was done with $20-35 \%$ collision energy (corresponding to about $10 \mathrm{~V}$ ). The default values for the other parameters were used directly without further optimizations for recording MS/MS spectra.

The key parts of the ND sampling interface are shown schematically in Fig. 1. Cheese products were sampled without any preparation. As shown in Fig. 1, a stream of neutral nitrogen gas at room temperature $\left(20^{\circ} \mathrm{C}\right)$ was used to vertically impact the cheese surface through a glass tube with a gas jet tip for ND sampling. Confined by the narrow diameter of the jet tip, the flow of gas is strong enough to desorb compounds from the cheese surface. A bell-shaped glass envelope (diameter $30 \mathrm{~mm}$, length $8 \mathrm{~cm}$; inset in Fig. 1) was fabricated to cover the sample airtightly to form 
Table 1 Basic information about the cheese samples

\begin{tabular}{|c|c|c|c|c|c|}
\hline Cheese type & Distributor & Number of samples & Hardness & Color & Milk source \\
\hline \multirow[t]{3}{*}{ Saint Paulin } & Migros & 3 & \multirow[t]{3}{*}{ Semisoft } & \multirow[t]{3}{*}{ Orange } & \multirow[t]{3}{*}{ Pasteurized cow's milk } \\
\hline & Coop & 5 & & & \\
\hline & Denner & 4 & & & \\
\hline \multirow[t]{3}{*}{ Emmentaler } & Migros & 3 & \multirow[t]{3}{*}{ Medium-hard } & \multirow[t]{3}{*}{ Yellow } & \multirow[t]{3}{*}{ Raw cow's milk } \\
\hline & Coop & 5 & & & \\
\hline & Denner & 7 & & & \\
\hline \multirow[t]{2}{*}{ Tilsiter } & Migros & 3 & \multirow[t]{2}{*}{ Semisoft } & \multirow[t]{2}{*}{ Light yellow } & \multirow[t]{2}{*}{ Pasteurized cow's milk } \\
\hline & Coop & 8 & & & \\
\hline \multirow[t]{3}{*}{ Gruyère } & Migros & 4 & \multirow[t]{3}{*}{ Hard } & \multirow[t]{3}{*}{ Yellow } & \multirow[t]{3}{*}{ Raw cow's milk } \\
\hline & Coop & 2 & & & \\
\hline & Denner & 5 & & & \\
\hline
\end{tabular}

All the samples were produced from cow's milk in Switzerland.

an aerosol which carries the neutral constituents from the sample surface. The neutral aerosol was collected by the glass envelope and flowed out through the only air tap. The desorbed analytes were introduced via this aerosol flow into the EESI source through a sample transfer line. The setup was designed to completely isolate the high-voltage probe from the samples, providing a high degree of operational safety of the device.

Optimization was done by adjusting individual experimental parameters. The following parameters were chosen for experiments: the distance between the neutral gas jet tip and the surface of the analytes was $2-3 \mathrm{~mm}$; the sampling area was estimated to be about $10 \mathrm{~mm}^{2}$, which was fully covered by the ND sampling probe. A sharp jet (inner diameter $0.1 \mathrm{~mm}$ ) was formed at the end of the sheath gas line so that a high gas speed (nitrogen, about $300 \mathrm{~m} / \mathrm{s}$ ) was created using a gas flow of $200 \mathrm{~mL} / \mathrm{min}$. Analytes present on the cheese sample surface were liberated during the ND process and then transferred to the EESI source. After ionization, the ions were guided into the QTOF mass spectrometer for mass analysis. To produce primary ions with high efficiency, a methanol/acetic acid/water solution $(45: 10: 45 ; \mathrm{v} / \mathrm{v} / \mathrm{v})$ was directly infused at $2 \mu \mathrm{L} / \mathrm{min}$ into the ESI spray source by a syringe pump. A voltage of $-3.5 \mathrm{kV}$ was applied to the ESI needle. The experiments were carried out in negative ion mode. The default values of other parameters were directly used as advised by the instrument manufacturer. No further optimizations were performed.

In this experimental procedure, no time-consuming steps were needed except for sample loading and data acquisi-
Fig. 1 Atmospheric pressure neutral desorption (ND) extractive electrospray ionization $(E E S I)$ mass spectrometry (MS): the neutral molecular mixture from the surfaces of cheese products is desorbed without sample pretreatment by a nitrogen gas flow at room temperature. The ND device was modified from the top part of a gas wash bottle, which covered the sampling area in an airtight manner, to sample the analytes into the EESI source. $M S$ mass spectrometer, QTOF quadruple time of flight

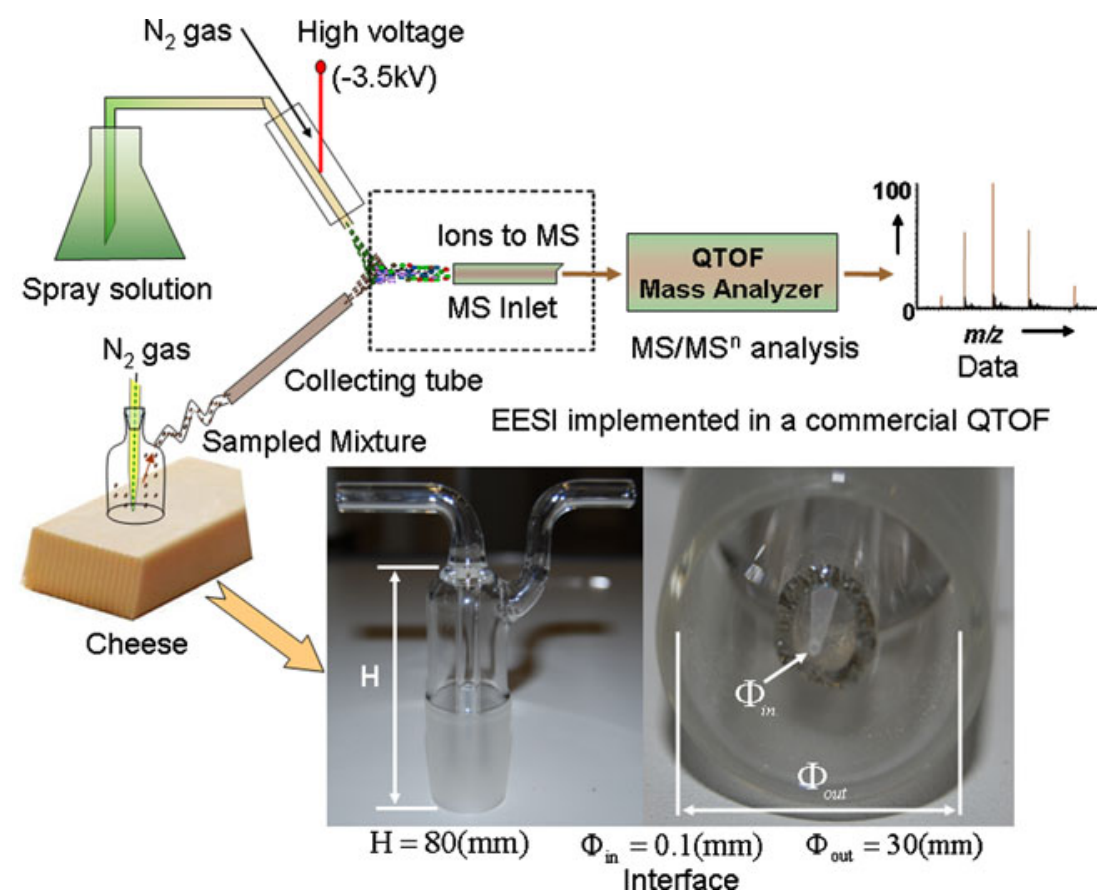


tion. Mass-spectral data were averaged over 100 scans, which took only $50 \mathrm{~s}$ in total. The high-speed data acquisition (twice a second) and the absence of any need for sample pretreatment should in the future allow this technique to perform real-time high-throughput analyses of cheeses, for example, by using an automated sample introduction system [30].

\section{Processing of mass-spectral data}

The raw mass spectra were exported manually as .txt files. All data were arranged by using the $\mathrm{m} / \mathrm{z}$ values as the independent variables and the absolute signal intensities as the dependent variables. All the mass spectra were imported into MATLAB (version 7.0, Eigenvector Research, Manson, WA, USA) and were treated as a matrix. Some data pretreatment (autoscaling) [15] was performed using the "auto" function in PLS_Toolbox 2.1 (for use with MATLAB, Eigenvector Research) to homogenize the peak intensities. The autoscaled data matrix was finally imported into commercial multivariate statistical analysis software (STATISTICA, version 6.0, StatSoft, Tulsa, OK, USA) spreadsheets (.sta files) for principal component analysis (PCA) evaluation. Once all the data had been loaded successfully into STATISTICA, PCA was executed without any further data treatment. After PCA had been performed, scores of the second, third, and fourth principal components (PCs) were selected for modeling.

\section{Results and discussion}

Effects of gas flow rate on chemical fingerprints

Cheese products contain various fatty acids [31, 32]. As mentioned already, the negative ion mode was chosen for EESI-MS to obtain characteristic mass fingerprints. In this study, the gas flow rate was investigated in view of its influence on the signals. Chemical fingerprints of the same spot of one cheese slice (Emmentaler from Denner) were recorded with different flow rates $(100,150$, and $200 \mathrm{~mL} / \mathrm{min}$ ). The results are shown in Fig. 2. Deprotonated molecular ion peaks at $\mathrm{m} / \mathrm{z} 87$ and 101 dominated the spectra at all flow rates (Fig. 2). These signals correspond to common semivolatile compounds in the cheese samples, butyric acid $(\mathrm{m} / \mathrm{z} 87)$ and valeric acid $(\mathrm{m} / \mathrm{z}$ 101). The remarkably high intensities are attributed to the high concentration and/or volatility of these analytes, which made them very easy to detect at all flow rates used. Several other peaks, such as $m / z 73$ (2-butanol), began to appear with relatively low intensities (249 cps) when the flow rate was raised to $150 \mathrm{~mL} / \mathrm{min}$ (Fig. 2b),
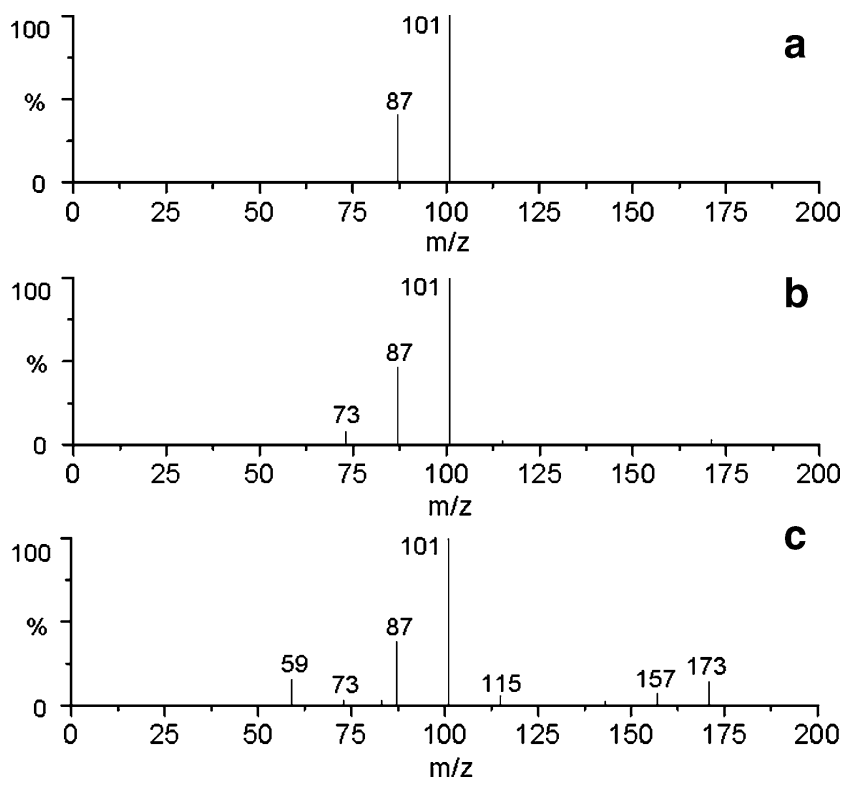

Fig. 2 Chemical fingerprints of the same spot of one cheese slice (Emmentaler from Denner), recorded in the negative ion mode with different flow rates by ND-EESI-QTOF-MS: a gas flow rate of $100 \mathrm{~mL} / \mathrm{min}$; b gas flow rate of $150 \mathrm{~mL} / \mathrm{min}$; $\mathbf{c}$ gas flow rate of $200 \mathrm{~mL} / \mathrm{min}$

and many more peaks (such as $m / z 59,115,157$, and 173) were detected with relatively high intensities $(986,281$, 388 , and $745 \mathrm{cps}$, respectively) at a flow rate of $200 \mathrm{~mL} /$ min. Since the data in Fig. 2 were recorded at the same spot, we interpret this finding to be due to liberation of compounds that had not yet been depleted from deeper inside the sample matrix by the higher nitrogen flow. As a general trend, the higher the flow rate, the more peaks could be observed. However, starting from a certain value (about $1 \mathrm{~L} / \mathrm{min}$ ), further increase of the nitrogen flow rate resulted in a decrease of the signals. In the extreme case, when the flow rate was higher than $3 \mathrm{~L} / \mathrm{min}$, no more peaks were observed in the mass spectra. This is probably caused by the inefficient ionization due to dilution and a very short residence time of the desorbed neutrals inside the ionizing plume. Disappearance of the signals was also observed when the gas flow rate was lower than $100 \mathrm{~mL} / \mathrm{min}$. This was because the weak nitrogen gas flow could not release analytes from the sample surface, especially nonvolatile substances. The same phenomenon was also observed in other EESI applications [18, 33]. A gradual fading of the signals was observed when the gas flow remained on one spot for several minutes $(\approx 10 \mathrm{~min})$ even with a gas flow of only 1 $\mathrm{L} / \mathrm{min}$.

In our experiments, the optimum gas flow was found to be $200 \mathrm{~mL} / \mathrm{min}$. Unless otherwise noted, all mass spectra in this study were recorded at this flow rate. 
Signal reproducibility and sample heterogeneity

The reproducibility of the experimental results is very important for data analysis, especially when chemometric approaches are applied. Very reproducible results are easily obtained if the experimental parameters are precisely adjusted and fixed. The reproducibility of ND-EESI-MS for cheese analysis was investigated by measuring the same spot of one cheese slice (Emmentaler from Denner) twice. The results are shown in Fig. 3a and b. Obviously, there are only very minor intensity variations and almost no variations in chemical composition. The correlation coefficient of the mass spectra in Fig. 3a and b was 0.9959. In Fig. $3 \mathrm{a}$ and $\mathrm{b}$, signals with significant intensities were mainly in the range between 50 and $300 \mathrm{Da}$. No peaks above $m / z 300$ were observed. This is perhaps because molecules with higher molecular weights have higher affinities for the cheese matrix, are probably less volatile, and are thus more difficult to desorb. This is consistent with a previous study in which perfumes was classified by NDEESI-MS [34]

The mass-spectral data appear quite simple despite the fact that cheese is known to have a very complex composition. Blending and homogenization are carried out during cheese manufacturing, yet the heterogeneity of cheese samples is still one of the main parameters that needs to be understood for successful characterization by EESI-MS. Another spot on the same piece of cheese (Emmentaler from Denner), which was a bit darker than the one characterized in Fig. 3a, was subjected to EESI-MS analysis in the same way (shown in Fig. 3e). Several dark spots were randomly distributed over the surface of this piece of cheese. The differences in both peak intensities and chemical composition were much more pronounced when comparing Fig. 3a and e than when comparing Fig. 3a and b. For example, some new peaks appeared (e.g., $m / z 60,89$, 199, and 212), whereas some peaks disappeared (e.g., $\mathrm{m} / \mathrm{z}$ 87,227 , and 255). The peak at $m / z 171$ had the maximal relative intensity in the spectrum in Fig. 3e, rather than $\mathrm{m} / \mathrm{z}$ 101 in Fig. 3a and b. Apparently, the surface quality did have some effect on the ND efficiency and on the release of compounds from the cheese surface. Care was thus taken to always sample parts of the surface that looked similar. Chemical fingerprints of two different spots with similar color on another type of cheese (Tilsiter from Coop) were also investigated (Fig. 3c, d). The chemical fingerprints of different spots only showed very slight variations of the relative peak intensities. One noticeable variation, as can be seen in Fig. 3c, was that the relative intensity of the peak at $m / z 101$ was higher than that of $m / z 171$ in Fig. 3d. These
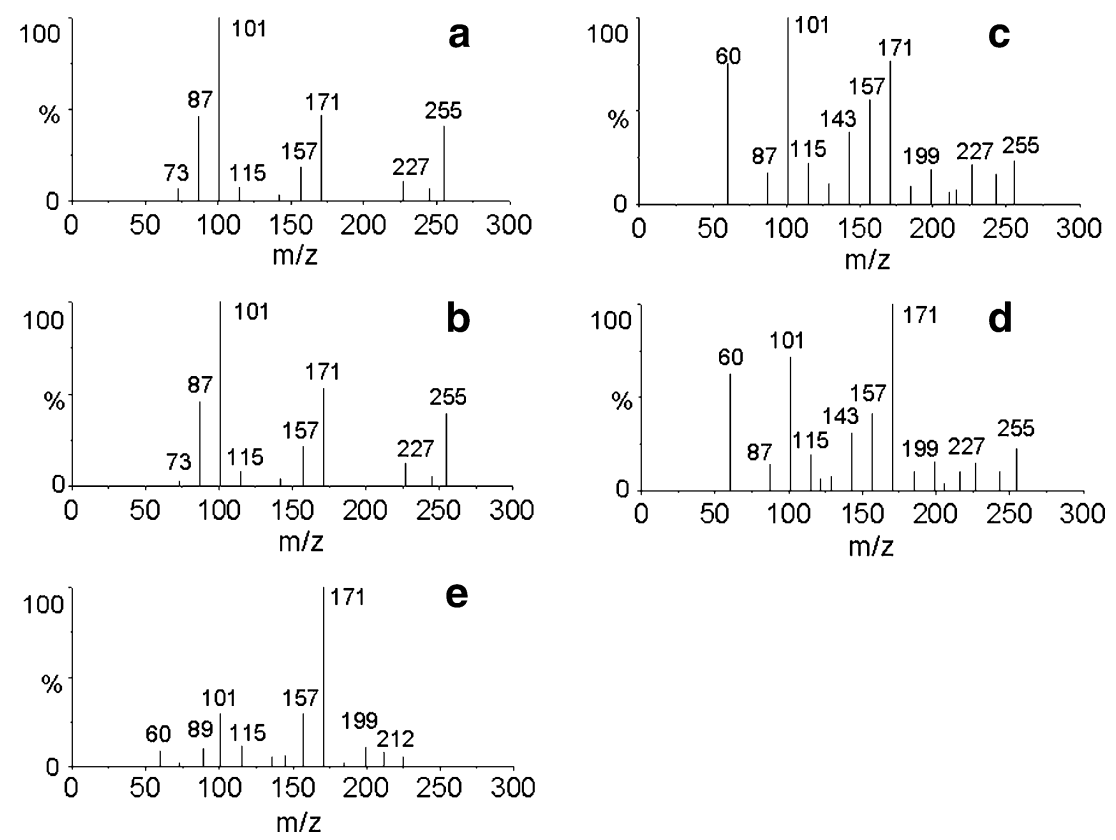

Fig. 3 Investigation of the repeatability of the ND-EESI-MS method and homogeneity of the sample: a mass-spectral fingerprint of one spot of the cheese slice (Emmentaler from Denner) in negative ion mode; $\mathbf{b}$ repeat measurement on the same spot as in a with the same working parameters; c mass-spectral fingerprint of one spot of another type of cheese product (Tilsiter from Coop) in negative ion mode; $\mathbf{d}$ mass-spectral fingerprint of another new spot of the same sample as in

c with the same working parameters; e mass-spectral fingerprint of a different spot of the same cheese sample as in a-the spot was similar to the others, but was a bit darker. The results of repeated measurements indicated that the accuracy and repeatability are high. The spots with different appearance show significant differences in the chemical composition and peak intensities. The chemical fingerprints of different spots show some variations of peak ratios 
findings suggest that the detectable chemical constituents of the cheese products are generally uniformly distributed through the whole sample. Overall, the spectrum of a random spot with a morphology similar to other parts of the sample surface was well representative for all parts of these cheese samples; the heterogeneity of our cheese samples was acceptable for further investigations.

\section{Representative MS/MS mass spectra}

Compounds of interest detected using ND-EESI-MS can be identified by using multiple-stage MS. As an example, the peak at $m / z 171$ was subjected to tandem MS experiments using CID for structure elucidation. As shown in Fig. 4, the deprotonated molecule generated major fragments under mild CID conditions ( $20 \%$ collision energy, $30 \mathrm{~ms})$ by the neutral loss of 44 and 46 mass units, which most likely corresponded to $\mathrm{CO}_{2}$ and $\mathrm{HCOOH}$, respectively. The intensity of the fragment ion peak at $m / z 125$ was much higher than that of the peak at $m / z$ 127. This useful information allows the deprotonated molecular ion to be tentatively interpreted as decanoic acid (a nonvolatile fatty acid; inset in Fig. 4), which is known to be formed during the ripening process [35]. A similar fragmentation pattern was also obtained with an authentic decanoic acid sample.

\section{Chemical fingerprints of cheese samples}

Chemical fingerprints of all the cheese samples were recorded using optimized experimental parameters. The information-rich mass spectra (Fig. S1) indicate the powerful desorption capacity of ND-EESI-MS. The massspectral fingerprints of Saint Paulin (Fig. S1i) and Tilsiter (Fig. S1k) showed abundant peaks, with $m / z 171$ exhibiting maximum relative intensity. Although the peaks were almost the same in these two cheese types, their relative abundances were very different. For example, the peak at $\mathrm{m} / \mathrm{z} 101 \mathrm{had}$ the second highest signal intensity in Tilsiter but was very weak in Saint Paulin. On the other hand, for

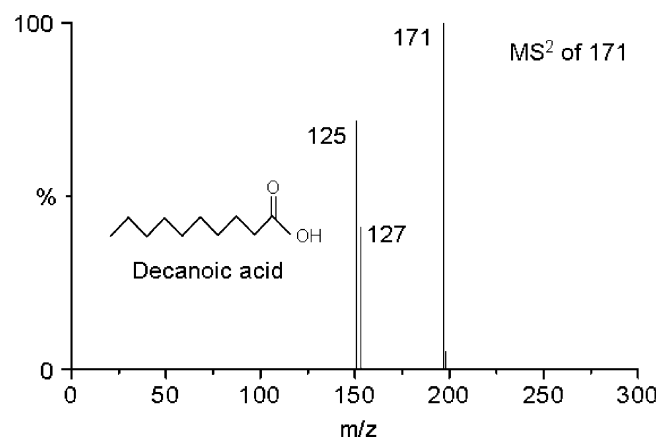

Fig. 4 MS/MS spectrum of the predominant peak at $\mathrm{m} / \mathrm{z} 171$ recorded using ND-EESI-QTOF-MS the chemical fingerprint of Emmentaler (Fig. S1c) and Gruyère (Fig. S1f), fewer strong peaks were found. The peak at $m / z 73$ had the highest relative intensity in Emmentaler, with other peaks being of much lower intensity. The spectral pattern of Gruyère was quite similar to that of Emmentaler. Differences in the chemical fingerprints among various types were obvious and reproducible.

There are some differences in peak intensities and chemical constituents among samples of the same cheese type but from different distributors, although these appear to be less significant than the differences between different types. For example, the most abundant peaks in Gruyère from various distributors were detected at $\mathrm{m} / \mathrm{z}$ 115 (Denner), $m / z 171$ (Coop), and $m / z 87$ (Migros), respectively. Other detectable chemical constituents also showed different abundances. There were even peaks which were undetectable in some of the samples, whereas they were easily found in samples of the same type but from another distributor. Typical examples are the signals at $m / z 60$ in Emmentaler and $m / z 87$ in Saint Paulin. We suppose the reason might be that the same type of cheese purchased from different distributors may originate from various manufacturers (or producing areas). In other words, the primary criterion for differentiation should be the cheese type, perhaps a secondary criterion should be the precise origin of the sample (if it can be identified, which is often not the case), but certainly not the identity of the distributor.

Among all the mass-spectral fingerprints (Fig. S1), peaks at $m / z 115,143,157$, and 171 were present in all samples, whereas some other deprotonated molecules $(\mathrm{m} / \mathrm{z} 60,73$, and 89) were only detected in some cheese samples. The

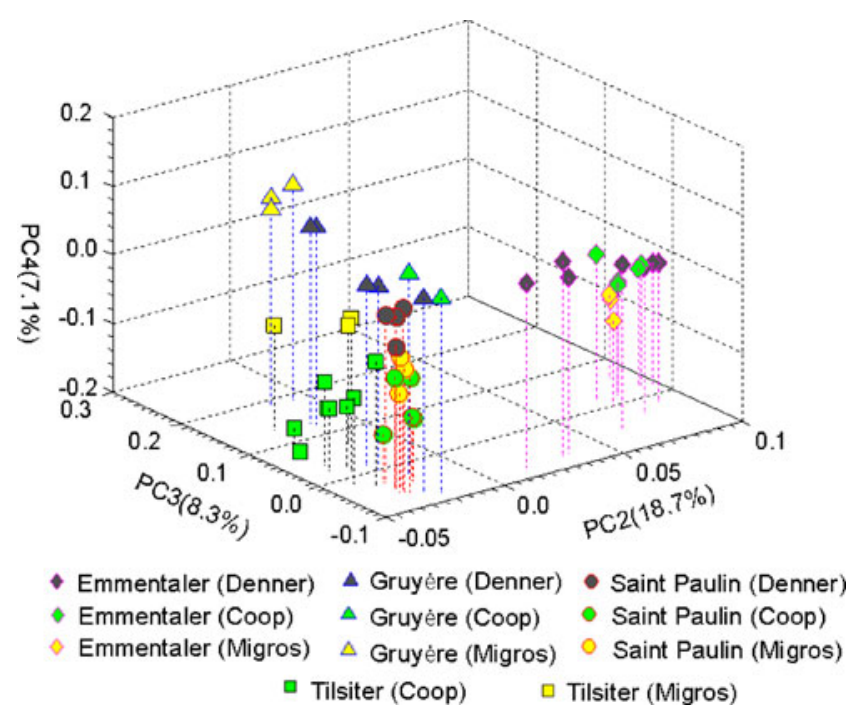

Fig. 5 Three-dimensional score plots of the principal component $(P C)$ analysis result for different types from various distributors of all the cheese products 
variation of peak intensity was the most remarkable character, even though the chemical compositions also varied to some degree.

\section{Differentiation of cheese samples by PCA}

All data from the cheeses investigated (four types from three distributors, 49 individual samples overall) were treated as one matrix (autoscaled by MATLAB 7.0) and analyzed by PCA to elucidate the effect of each influencing factor (i.e., type, distributor, and sensory properties) on the classification performances. PC1 gave only an ambiguous separation for all influencing factors, so it was not used in the model although it represented $53.1 \%$ of the total variance. This is probably because that loading of PC1 contains signals of common major compounds present in all cheese samples. A 3D graph of the PCA scores (Fig. 5) showed a successful separation in the direction of PC2, PC3, and PC4. These PCs showed the best distinguishing ability according to type, since none of the data points of the same type overlapped in this $3 \mathrm{D}$ plot, which represented $34.1 \%$ of the total variances. This means that these PCs exactly described the variations of different cheese types in the measurements. The percentages of variance explained by PC2, PC3, and PC4 were 18.7, 8.3, and $7.1 \%$, respectively. Confident differentiation of samples underscored the ability of ND-EESI-MS to discriminate the various types of cheeses. Remarkably, all samples of Emmertaler uniquely grouped within the positive range of PC2. This result indicates that cheese samples of this type showed greater differences in the detected components and their concentration distribution compared with the others. The results were in good agreement with mass-spectral fingerprint features from Emmertaler, because fewer peaks were detected in Emmertaler cheeses from all distributors (Fig. S1a-c) compared with the other types. The Emmertaler from Denner (marked with a gray color) was distributed over a larger interval which overlapped with data points of the Coop (green color) and Migros (yellow color) samples of Emmertaler, which were narrowly distributed. This distribution indicates that the PCA was unable to tell the differences among various distributors. This is quite understandable since the distributors have little to do with the quality of cheeses. On the other hand, although the data points of the other three types are located in a narrow interval on the PC2 axis, there were no serious overlaps.

It should also be noted that apparently the ND-EESI data are insensitive to other physical qualities (such as color or hardness) and preprocessing of cow's milk (raw vs. pasteurized milk) of these cheeses. These features were undistinguishable in the 3D graph of PCA scores (Fig. 5), although many factors (i.e., milk origin, milk treatment, season, manufacturing conditions, and ripening time and temperature), as reported above, can affect the qualities of cheese products to a certain degree. The difference in cheese type, as shown from the PCA results, is the dominating factor for the successful discrimination of cheese type. The strong peaks of deprotonated molecules at $m / z$ 60, 73, 87, 101, 115, and 171 were the most predominant ions in the loadings of $\mathrm{PC} 2, \mathrm{PC} 3$, and $\mathrm{PC} 4$ (Fig. S2) which made great a contribution to distinguishing the results. Those constituents might be used as biomarkers for discriminating cheese types.

\section{Conclusions}

In this study, the concept of ND sampling was extended to greasy sample surfaces for the first time. Both volatile analytes (such as butyric acid and valeric acid) and nonvolatile analytes (such as decanoic acid) could be directly sampled by a ND gas beam. Our results also show that ND-EESI-MS combined with PCA is a powerful analytical tool for high-throughput differentiation of cheeses of different types, providing high sensitivity and a high information content. This makes ND-EESI-MS a possible choice for high-throughput, real-time, and online analysis of biological samples in various complex matrices. These figures of merits are attractive for commercial trade and safety management, particularly in cases where unsatisfactory results are possibly generated by other methods such as morphological characteristics analysis and sensory measurements.

Acknowledgements This work was jointly supported by grants from MOST of China (grant no. 2009DFA41880, to H.C.) and by the Sino-Swiss Science and Technology Cooperation (SSSTC, project no. IZL CZ2 123987, to R.Z.).

\section{References}

1. Chan EYY, Griffiths SM, Chan CW (2008) Lancet 372:14441445

2. Kleter GA, Marvin HJP (2009) Food Chem Toxicol 47:10221039

3. Pillonel L, Bosset JO, Tabacchi R (2002) Lebensm Wiss Technol $35: 1-14$

4. Gambelli L, Manzi P, Panfili G, Vivanti V, Pizzoferrato L (1999) Food Chem 66:353-358

5. Merdivanb M, Yilmaz E, Hamamci C, Aygunc RS (2004) Food Chem 87:163-171

6. Papademas P, Robinson RK (2000) Int Dairy J 10:762-768

7. Bugaud C, Buchin S, Noël Y, Tessier L, Pochet S, Martin B, Chamba JF (2001) Lait 81:593-607

8. Cagno RD, Buchin S, Candia SD, Angelis MD, Fox PF, Gobbetti MJ (2007) Dairy Sci 90:2689-2704

9. Zellner BDA, Dugo P, Dugo G, Mondello L (2008) J Chromatogr A $1186: 123-143$

10. Schaller E, Bosset JO, Escher F (1999) Chimia 53:98-102 
11. Vaclavik L, Cajka T, Hrbek V, Hajslova (2009) J Anal Chim Acta 645:56-63

12. Sampson JS, Hawkridge AM, Muddiman DC (2006) J Am Soc Mass Spectrom 17:1712-1716

13. Takáts ZNM, Wiseman J, Gologan B, Cooks RG (2004) Science 306:471-473

14. Yang SP, Han J, Huan YF, Cui YJ, Zhang X, Chen HW, Gu HW (2009) Anal Chem 81:6070-6079

15. Wu ZC, Chen HW, Wang WL, Jia B, Yang TL, Zhao ZF, Ding JH, Xiao XX (2009) J Agric Food Chem 57:9356-9364

16. Chen HW, Zheng J, Zhang X, Luo MB, Wang ZC, Qiao XL (2007) J Mass Spectrom 42:1045-1056

17. Chen HW, Wortmann A, Zhang WH, Zenobi R (2007) Angew Chem Int Ed 46(4):580-583

18. Chen HW, Sun YP, Wortmann A, Gu HW, Zenobi R (2007) Anal Chem 79(4):1447-1455

19. Li M, Hu B, Li JQ, Chen R, Zhang X, Chen HW (2009) Anal Chem 81:7724-7731

20. Chen HW, Zenobi R (2008) Nat Protoc 3(9):1467-1475

21. Ding JH, Gu HW, Yang SP, Li M, Li JQ, Chen HW (2009) Anal Chem 81:8632-8638

22. Chen HW, Hu B, Hu Y, Huan YF, Zhou ZQ, Qiao XL (2009) J Am Soc Mass Spectrom 20:719-722
23. Wu D, Feng S, He Y (2008) J Dairy Sci 91(3):939-949

24. Liu F, He Y (2008) Food Res Int 41:562-567

25. Chong XM, Hu CQ, Feng CY, Pang HH (2009) Vib Spectrosc 49:196-203

26. Luo DH, Hosseini HG, Stewart JR (2004) Sens Actuators B 99:253-257

27. Khatib A, Wilson EG, Kim HK, Lefeber AWM, Erkelens C, Choi YH, Verpoorte R (2006) Anal Chim Acta 559:264-270

28. Manca G, Franco MA, Versini G, Camin F, Rossmann A, Tola A (2006) J Dairy Sci 89:831-839

29. Aprea E, Biasioli F, Gasperi F, Mott D, Marini F, Mark TD (2007) Int Dairy J 17:226-234

30. Chen HW, Talaty NN, Takáts ZN, Cooks RG (2005) Anal Chem 77:6915-6927

31. Upreti P, Metzger LE (2006) J Dairy Sci 89:420-428

32. Metzger LE, Barbano DM, Rudan MA, Kindstedt PS (2000) J Dairy Sci 83:648-658

33. Chingin K, Chen HW, Gamez G, Zhu L, Zenobi R (2009) Anal Chem 81:123-129

34. Chingin K, Gamez G, Chen HW, Zhu L, Zenobi R (2008) Rapid Commun Mass Spectrom 22:2009-2014

35. Mulet A, Escriche I, Rossello C, Tarrazó J (1999) Food Chem $65: 219-225$ 